\title{
Preface to the special issue on Security in global computing
}

Published online: 9 June 2004 - (c) Springer-Verlag 2004

Global computing is an emerging area of research that is attracting increasing attention by a large community of researchers from around the world. The main characteristics of global computing applications are as follows.

- The systems are composed of autonomous computational entities where activity is not centrally controlled, either because global control is impossible or impractical or because the entities are controlled by different owners.

- The computational entities are mobile due to the movement of the physical platforms or by movement of the entity from one platform to another.

- The configuration varies over time. For instance, the system is open to the introduction of new computational entities and likewise their deletion. The behavior of the entities may vary over time.

- The systems operate with incomplete information about the environment. For instance, information becomes rapidly outdated and mobility requires information about the environment to be discovered.

The ultimate goal of the burgeoning research activity is to provide a solid scientific foundation for the design of such systems and to lay the groundwork for achiev- ing effective principles for building and analyzing such systems.

The description above makes it immediately apparent that one of the key points of global computing is the handling of information security.

This special issue collects extended versions of papers presented at the international workshop on global computing held in Rovereto, Italy, 9-14 February 2003.

The first paper of the issue, by Teller, Zimmer, and Hirschkoff, shows how usage of resources can be made secure in the setting of mobile computations.

The second contribution, by Buchholtz, Riis Nielson, and Nielson, introduces control flow analysis as a mechanism to study the security of communication protocols.

Gorrieri, Lanotte, Maggiolo-Schettini, Martinelli, Tini, and Tronci define timed security and present a tradeoff between time properties and security properties.

The last contribution, by Bartoletti, Degano, and Ferrari, reports the results of a study on the security issues of access control in Java by developing a static stackinspection technique.

Corrado Priami

Università di Trento

March 2004 